\title{
Outcome of medium chain acyl-CoA dehydrogenase deficiency after diagnosis
}

\author{
Callum J Wilson, Michael P Champion, Jane E Collins, Peter T Clayton, James V Leonard
}

\begin{abstract}
Background-Medium chain acyl-CoA dehydrogenase (MCAD) deficiency is the most common inborn error of fatty acid metabolism. Undiagnosed, it has a mortality rate of $20-25 \%$. Neonatal screening for the disorder is now possible but it is not known whether this would alter the prognosis.

Objective-To investigate the outcome of MCAD deficiency after the diagnosis has been established.

Method-All patients with a proved diagnosis of MCAD deficiency attending one centre in a four year period were reviewed.

Results-Forty one patients were identified. Follow up was for a median of 6.7 years (range, 9 months to 14 years). Nearly half of the patients were admitted to hospital with symptoms characteristic of MCAD deficiency before the correct diagnosis was made. After diagnosis, two patients were admitted to hospital with severe encephalopathy but there were no additional deaths or appreciable morbidity. There was a high incidence (about one fifth) of previous sibling deaths among the cohort.

Conclusions-Undiagnosed, MCAD deficiency results in considerable mortality and morbidity. However, current management improves outcome, supporting the view that the disorder should be included in newborn screening programmes. (Arch Dis Child 1999;80:459-462)
\end{abstract}

Keywords: medium chain acyl-CoA dehydrogenase deficiency; outcome; screening; carnitine; inborn errors of metabolism

Metabolic Unit, Great Ormond Street Hospital for Children, Great Ormond Street, London WC1N 3JH, UK

C J Wilson

M P Champion

J E Collins

Biochemistry, Endocrinology and Metabolism Unit, Institute of Child Health, London, UK P T Clayton

J V Leonard

Correspondence to: Dr Wilson. email: callumjwilson $a$ yahoo.com

Accepted 22 December 1998

Medium chain acyl-CoA dehydrogenase (MCAD) deficiency is the most common inborn error of fatty acid metabolism, ${ }^{1}$ with a prevalence of up to 1 in 10000 in some areas of the UK. ${ }^{2}$ It usually presents in early childhood with hypoglycaemia and encephalopathy after an intercurrent illness and/or a period of poor oral intake. ${ }^{13-5}$ There is a high risk of death or permanent neurological sequelae with the attacks. ${ }^{3-6}$ The development of tandem mass spectrometry enables a rapid diagnosis to be made using a blood spot (Guthrie card) so that universal neonatal screening for the condition is now feasible. ${ }^{78}$ However, a screening test is only justified if effective treatment is available. ${ }^{9}$ Although treatment is said to work well, no studies have looked specifically at the course of the illness after the diagnosis has been made. Our study was designed to do just this.

\section{Methods}

After the publication of the management of intercurrent illness, ${ }^{10}$ we standardised our treatment of MCAD deficiency. We studied all children with MCAD deficiency seen either acutely, or as an outpatient, by members of the metabolic units at Great Ormond Street and Guy's Hospital during the period September 1993 to September 1997.

We reviewed the clinical notes of these patients. We recorded the number and severity of episodes of decompensation that the child had suffered, both before and after the diagnosis of MCAD deficiency. For our study, the severity of the each episode was confined to there being either: (1) coma or (2) lethargy/ hypoglycaemia (before diagnosis) or lethargy/ hypoglycaemia/intercurrent illness (after diagnosis). In addition, we compared the child's physical and cognitive state three months after the initial diagnosis with that noted at the time of their most recent review. We contacted those patients who had not been seen in the outpatient clinic over the last 12 months and their parent(s) were interviewed directly. We based our cognitive evaluation on the paediatrician's assessment of the child and on the completion of a structured questionnaire.

\section{Results}

THE PATIENTS

Forty one patients met the criteria listed above and were included in our study.

We identified eight children prospectively after the diagnosis of a sibling. Five were neonates and the remaining three were aged 23 months, 3 years, and 15 years.

The other 33 patients presented acutely; 12 of them during our study period and the remaining 21 before September 1993. All were seen in the outpatient department during our study period. In 29 of these 33 cases, MCAD deficiency was diagnosed after the child had suffered a brief intercurrent illness, associated with a period of anorexia, and often an overnight fast. They had become lethargic and hypoglycaemic and in two thirds of cases were unconscious at presentation. Two patients, despite having been admitted to hospital with symptoms characteristic of MCAD deficiency, were only diagnosed after a sibling was confirmed to have the disorder. One patient was diagnosed after investigation for recurrent seizures and developmental delay and another after investigations for mild dysmorphic features at birth. The median age of diagnosis of 
Table 1 Hospital admissions before and after diagnosis of MCAD deficiency

\begin{tabular}{|c|c|c|c|c|c|c|}
\hline \multirow[b]{2}{*}{ Initial diagnosis } & \multicolumn{3}{|c|}{$\begin{array}{l}\text { Admissions before diagnosis (excluding the } \\
\text { admission that led to the diagnosis) }\end{array}$} & \multicolumn{3}{|c|}{ Admissions after diagnosis } \\
\hline & Coma & $\begin{array}{l}\text { Lethargy/ } \\
\text { hypoglycaemia }\end{array}$ & No admissions & Coma & $\begin{array}{l}\text { Intercurrent } \\
\text { illness/lethargy }\end{array}$ & No admission \\
\hline Prospective $(\mathrm{n}=8)$ & & & 8 & 0 & $2^{\star}$ & 6 \\
\hline Symptomatic $(\mathrm{n}=33) \dagger$ & $8 \ddagger$ & $9 \$$ & 16 & $2 \pi$ & $10^{\star \star}$ & 20 \\
\hline Total $(\mathrm{n}=41) \dagger$ & 8 & 9 & 24 & 2 & 12 & 26 \\
\hline
\end{tabular}

^Both had more than three admissions.

†Because one patient died during the initial admission, $n=32$ and $n=40$, respectively, in the follow up analysis.

$\ddagger$ Three of these patients had more than one episode of coma. Two others had also been admitted with lethargy.

$§ S i x$ of these patients had more than one episode.

TOne of these patients had numerous admissions, most of them were more suggestive of febrile convulsions rather than MCAD related metabolic decompensation.

$\star \star$ Five of these patients had more than three admissions.

these 33 patients was 18 months (range, 6 weeks to 10.5 years). A diagnostic fast was needed to secure the diagnosis in eight patients.

There were five pairs of siblings in the cohort; one in each pair was diagnosed prospectively.

DIAGNOSIS

We confirmed the diagnosis in all patients by measuring acyl carnitines by means of electrospray ionisation tandem mass spectrometry. ${ }^{8}$

In addition, 36 patients were homozygous for the A985G mutation. A further three patients were heterozygous for the A985G mutation. All these patients not only had characteristic acyl carnitines but also typical urinary organic acids measured by gas chromatography/mass spectrometry and were presumed to be compound heterozygotes. The two patients in whom genotyping was not performed were siblings. Both had characteristic urinary organic acids and acyl carnitines.

DECOMPENSATIONS BEFORE DIAGNOSIS

Excluding the five patients diagnosed prospectively as neonates, 17 of 36 cases in our cohort had been admitted to hospital with symptoms characteristic of MCAD deficiency before the admission that led to the correct diagnosis being made (table 1 ).

Eight patients had previous admissions to hospital with coma. Three of these had had more than one episode of coma whereas another two, in addition to the admission with coma, had been seen on separate occasions with lethargy and/or hypoglycaemia.

A further nine patients had previous admissions to hospital with lethargy and/or hypoglycaemia. Six of these nine patients had had more than one episode.

\section{INITIAL OUTCOME}

One patient died during the initial admission to hospital. Assessment of the remaining 30 symptomatic patients, three months after their discharge from hospital, revealed one to have mild cognitive impairment, two to have moderate impairment, and another two to have severe impairment. One patient in each of these last two groups had previously shown some cognitive impairment, having had previous episodes of severe decompensation.
TREATMENT

A clinician and a dietitian from our metabolic department saw all the patients and their families. The parents, the general practitioner, and the local paediatrician were given careful advice on the importance of avoidance of fasting and the use of regular high carbohydrate drinks during periods of infection and anorexia. Paediatricians were asked to give patients free access to the children's ward to ensure no delay in starting a glucose infusion if the child was unable to tolerate the "emergency regimen" drinks. ${ }^{10}$ Carnitine was not given routinely. Three patients, all of whom had had recurrent admissions to hospital, were later treated with carnitine.

DECOMPENSATIONS AFTER DIAGNOSIS

The length of follow up ranged from 10 months to 14 years, with a median of 6 years. Twelve patients required subsequent admission to hospital for observation and/or intravenous fluids during periods of intercurrent illness. Seven of these patients had more than three admissions.

Two patients were admitted with encephalopathic coma. One of these patients needed recurrent admissions. However, these were usually during a febrile illness, and were associated with a seizure, not hypoglycaemia, and were thus thought to have febrile convulsions. The relative contribution of the MCAD deficiency in this patient is uncertain.

Twenty six patients had no further admissions to hospital (table 1).

FINAL OUTCOME

From the time of diagnosis and initiation of treatment there were no deaths or appreciable additional cognitive impairment in our study group. One patient developed a mild hemiparesis secondary to the initial metabolic decompensation at the time of diagnosis.

One patient had a period of psychogenic visual loss, which later improved; another had severe feeding problems and required a nasogastric tube; and a third child developed severe behavioural problems.

SIBLINGS

There were 36 pedigrees in our study. Of the 42 siblings within these families, nine had died of an illness highly suggestive of MCAD deficiency (table 2). 
Table 2 Details of sibling deaths

\begin{tabular}{lll}
\hline Age & "Cause" of death & Proven MCAD \\
\hline 15 months & Found dead in bed after viral illness & Not tested \\
2.5 years & Viral pneumonia & Yes \\
9 months & Died after viral illness & Not tested \\
4 months & Sudden infant death syndrome & Not tested \\
3.5 years & Reye's syndrome & Not tested \\
18 months & Reye's syndrome & Not tested \\
7 years & Severe hypoglycaemia and encephalopathy; died in hospital & Yes \\
3 years & Reye's syndrome & Not tested \\
8 months & Found dead in bed after viral illness & Not tested \\
\hline
\end{tabular}

Discussion

A number of studies has already established the high mortality (20-25\%) and morbidity from the initial severe metabolic decompensation in previously undiagnosed cases of MCAD deficiency. ${ }^{3-6}$ However, our study was designed to assess the outcome of MCAD deficiency after the diagnosis has been established and treatment begun. This is important information because a relatively simple screening method is now available using the neonatal blood spot and tandem mass spectrometry. ${ }^{78}$ Although it has been suspected that children remain relatively healthy after the diagnosis has been made, no studies have looked specifically at this issue. Wilcken et al, in reviewing 20 cases seen over a 17 year period up until 1993, noted that of the 16 patients available to follow up, only two had had a serious life threatening episode after diagnosis. ${ }^{5}$ Iafolla et al, in a retrospective questionnaire review of patients who were referred to their diagnostic laboratory service, noted that of 97 patients known to have MCAD deficiency $71 \%$ had had no further clinical episodes. ${ }^{3}$ The remaining 29\% had had an average of three further episodes requiring admission. The mean length of follow up in this study was 2.6 years and no mention was made of the severity of these episodes or of any comparison between the events before and after diagnosis. In both studies, several patients were lost to follow up. Somewhat disturbingly, Ziadeh et al reported that of 12 infants identified as part of a neonatal screening programme, two subsequently died as a result of the condition. ${ }^{11}$ Both these deaths were probably preventable (E Naylor, personal communication, 1997).

In our study, all patients have done well since the diagnosis was made. It is possible, however, that those patients who were diagnosed, and subsequently died, before September 1993, are not represented here. Thus, the sample could be biased towards those cases that have done well. However, we are aware of only one child with known MCAD deficiency who was seen at the hospital and who subsequently died. ${ }^{12}$ This was in 1990, before our treatment was standardised, and there was doubt that the metabolic disorder was responsible. Since 1993, our management of MCAD deficiency has been standardised, with particular attention being paid to supplementary high energy feeds during periods of illness. ${ }^{10}$ Even allowing for this child, the evidence presented here does suggest that MCAD deficiency can be managed successfully with a relatively low risk of severe metabolic upset. Without a control group (which is obviously ethically impossible) it is difficult to prove that treatment is successful. However, we feel that the comparison of the same group of children before and after treatment is justified.

The most important aspect of the management is parent (patient) and doctor education. The need is stressed for regular feeds, supplemented by high energy carbohydrate drinks (the emergency regimen) during periods of illness, and the need to seek medical attention if these drinks are refused. Oral rehydration fluids are not adequate. If oral (or nasogastric) feeds are not possible then intravenous fluids (10\% dextrose) should be started without waiting for symptoms of decompensation to develop. ${ }^{10}{ }^{13}$ With this policy, it is not surprising that minor admissions to hospital were common in this cohort of children. However, it was reassuring that few patients progressed to develop severe encephalopathy, and there was no obvious cognitive or physical regression in the children studied during the period after diagnosis. However, because direct observations, parental questionnaires, and school reports were relied upon for these assessments, rather than psychometric evaluation, we might have been missed subtle impairments. A number of parents reported that although their child was doing fine academically, they did have some minor difficulty in physical activities. These problems were not progressive and were not apparent on routine outpatient clinical assessment.

Three patients developed psychological/ behavioural problems. Whether this was secondary to neurological damage sustained before or after diagnosis, or whether it was caused by the stress placed on the child and family in coping with a chronic illness is unclear. Certainly, it became apparent when reviewing the families that although most seemed to cope well, with very little disruption to the child or household, some appeared to have great difficulty in coming to terms with the diagnosis.

Based usually on single case reports, carnitine has been advocated in the treatment of MCAD deficiency to reduce acute decompensations. ${ }^{14-16}$ We have not used carnitine as first line treatment in MCAD deficiency. In our study, 37 of 40 patients were managed successfully without carnitine. Although three patients began carnitine treatment later on, usually as a result of frequent hospital admissions, we have no evidence, either objectively or subjectively, that it was beneficial. However, some preliminary studies show that carnitine might improve exercise tolerance (P J Lee et al, unpublished observations, 1997). Relatively little emphasis is placed on the low fat diet recommended by some authors. ${ }^{1}$

Most authors report a mortality rate during the initial decompensation of $20-25 \% .^{3-6}$ Often, children die before medical help is obtained. Both the hospitals in our study are tertiary referral centres and thus receive patients who have already been seen at their local hospital. The low mortality rate of one in 
41 reported here is a reflection of this. If the nine siblings who almost certainly died of MCAD deficiency are included in the cohort then the mortality rate is $20 \%$. This may be an artificially low mortality rate because it includes only those deaths that have been ascertained through a known sibling case. Alternatively, it might be an overestimate because studies of the prevalence suggest that an appreciable number of children with MCAD deficiency could be asymptomatic and thus undiagnosed. $^{26}$

Most reviews of sudden infant death syndrome (SIDS) suggest a low incidence of MCAD. ${ }^{17}{ }^{18}$ This is not surprising because deaths from MCAD deficiency usually follow a viral-type illness, often with associated gastroenteritis, and are thus not typical of "cot death". These deaths might still be documented as "Reye's syndrome" or "viral pneumonia" and subsequent siblings not investigated. ${ }^{14}$

Despite the greater medical awareness of the disorder, there are still a large number of patients who are admitted to hospital with the characteristic symptoms of MCAD deficiency yet are not diagnosed rapidly. The mortality rate from the disorder may be as high as $20 \%$. It is now possible to diagnosis MCAD deficiency on the tandem mass spectrometry analysis of a Guthrie blood spot. Alternatively, DNA analysis looking for the common A985G mutation is readily available. Therefore, it is not usually necessary for children to go forward to a formal fasting test. Based on our evidence, once the correct diagnosis is known an effective yet relatively inexpensive treatment is available. To prevent the considerable mortality and morbidity of this disease the implementation of neonatal screening should be seen as a priority.
1 Roe C, Coates P. Mitochondrial fatty acid oxidation disorders. In: Scriver C, Beaudet A, Sly W, Valle D, eds. The metabolic and molecular basis of

2 Seddon HR, Green A, Gray RGF, Leonard JV, Pollitt RJ. Regional variations in medium-chain acyl-CoA dehydrogenase deficiency [letter]. Lancet 1995;345:135-6.

3 Iafolla AK, Thompson RJ, Roe CR. Medium-chain acyl-CoA dehydrogenase deficiency: clinical course in 120 affected children. F Pediatr 1994;124:409-15.

4 Touma EH, Charpentier C. Medium-chain acyl-CoA dehydrogenase deficiency. Arch Dis Child 1992;67:142-5.

5 Wilcken B, Hammond J, Silink M. Mortality and morbidity in medium chain acyl coenzyme A dehydrogenase defiin medium chain acyl coenzyme A Dis Child 1994;70:410-12.

6 Pollitt RJ, Leonard JV. Prospective surveillance study of medium chain acyl-CoA dehydrogenase deficiency in the UK. Arch Dis Child 1998;79;116-19.

7 Van Hove JL, Zhang W, Kahler SG, et al. Medium-chain acyl-CoA dehydrogenase (MCAD) deficiency: diagnosis by acylcarnitine analysis in blood. Am F Hum Genet 1993;52: 958-66.

8 Clayton PT, Doig M, Ghafari S, et al. Screening for medium chain acyl-CoA dehydrogenase deficiency using tandem mass spectrometry. Arch Dis Child 1998;79:109-15.

9 Committee on the Fetus and Newborn, American Academy of Pediatrics. Criteria for screening of newborn infants for of Pediatrics. Criteria for screening of newborn
metabolic disease. Pediatrics 1965;35:499-501.

10 Dixon MA, Leonard JV. Intercurrent illness in inborn errors of intermediary metabolism. Arch Dis Child 1992;75:11519

11 Ziadeh R, Hoffman EP, Finegold DN, et al. Medium chain acyl-CoA dehydrogenase deficiency in Pennsylvania: neonatal screening shows high incidence and unexpected mutation frequency. Pediatr Res 1995;37:675-8.

12 Lee P, Leonard JV. Fatty infiltration in the liver in medium chain acyl Co-A dehydrogenase deficiency. Arch Dis Child 1991;66:727-8.

13 Morris AA, Leonard JV. Early recognition of metabolic decompensation. Arch Dis Child 1997;76:555-7.

14 Roe CR, Millington DS, Maltby DA, Kinnebrew P. Recognition of medium chain acyl Co-A dehydrogenase deficiency in asymptomatic siblings of children dying of sudden infant death or Reye's syndromes. F Pediatr 1986;108:13-18.

15 Chalmers R. Proceedings of the workshop on L-carnitine therapy in inborn errors of metabolism. BIMDG Newsletter 1996;11:2-12.

16 Roe CR, Millington DS, Maltby DA, et al. Diagnostic and therapeutic implications of medium chain acylcarnitines in medium chain acyl-CoA dehydrogenase deficiency. Pediatr Res 1985;19:459-66.

17 Arens R, Gozal D, Kain K, et al. Prevalence of medium chain acyl-coenzyme A dehydrogenase deficiency in the chain acyl-coenzyme A dehydrogenase deficiency in the

18 Dundar M, Lanyon WG, Connor JM. Scottish frequency of the common G985 mutation in the medium-chain acyl-CoA dehydrogenase (MCAD) gene and the role of MCAD deficiency in sudden infant death syndrome (SIDS). F Inherit Metab Dis 1993;16:991-3. 